\title{
CURVATURE CONDITIONS ON RIEMANNIAN MANIFOLDS WITH BROWNIAN HARMONICITY PROPERTIES
}

\author{
H. R. HUGHES
}

\begin{abstract}
The time and place that Brownian motion on a Riemannian manifold first exits a normal ball of radius $\epsilon$ is considered and a general procedure is given for computing asymptotic expansions, as $\epsilon$ decreases to zero, for joint moments of the first exit time and place random variables. It is proven that asymptotic versions of exit time and place distribution properties that hold on harmonic spaces are equivalent to certain curvature conditions for harmonic spaces. In particular, it is proven that an asymptotic mean value condition involving first exit place is equivalent to certain levels of curvature conditions for harmonic spaces depending on the order of the asymptotics. Also, it is proven that an asymptotic uncorrelated condition for first exit time and place is equivalent to weaker curvature conditions at corresponding orders of asymptotics.
\end{abstract}

\section{INTRODUCTION}

It is a problem of interest to determine what properties of a Riemannian manifold can be deduced from properties of Brownian motion on the manifold. We will establish that certain curvature conditions for the manifold are equivalent to properties of the distributions of the times and places that Brownian motion first exits from normal balls in the manifold.

Let $M$ be an $n$-dimensional Riemannian manifold. Let $B_{m}(\epsilon)$ denote the geodesic ball of radius $\epsilon$ about $m$ in $M$. Let $S_{m}(\epsilon)$ denote the boundary of $B_{m}(\epsilon)$. Let $\left(X_{t}\right)$ be Brownian motion on $B_{m}(\epsilon)$, started from $m$. The infinitesimal generator of $\left(X_{t}\right)$ is $\frac{1}{2} \Delta$, where $\Delta$ is the Laplace-Beltrami operator on functions. Let $T_{\epsilon}$ be the first exit time of $X_{t}$ from $B_{m}(\epsilon)$ :

$$
T_{\epsilon}=\inf \left\{t>0: d\left(m, X_{t}\right) \geq \epsilon\right\} .
$$

For simplicity, when there is no ambiguity, we will use $T$ for $T_{\epsilon}$.

We examine the marginal and joint distributions of the first exit time and place, $T_{\epsilon}$ and $X_{T_{\epsilon}}$. In particular, we consider properties involving these distributions that hold on harmonic spaces and establish curvature conditions that are equivalent to asymptotic versions of these properties as $\epsilon \downarrow 0$.

We do this by extending the perturbation method of [GP] and [L1]. We give a general technique by which any joint moment of $T_{\epsilon}$ and $X_{T_{\epsilon}}$ can be expanded in terms of curvature expressions and powers of $\epsilon$.

Received by the editors May 6, 1994.

1991 Mathematics Subject Classification. Primary 58G32; Secondary 53B20, $60 \mathrm{~J} 65$.

Key words and phrases. Brownian motion, harmonic space, curvature conditions, exit time, exit place.

(C) 1995 American Mathematical Society 
In the remainder of this section we give curvature conditions for harmonic spaces and state our results. In Section 2, we introduce the family of homogeneous harmonic polynomials which are useful working on the unit ball and unit sphere in $\mathbb{R}^{n}$. In Section 3, we describe how a perturbation method can be used to expand joint moments of $T$ and $X_{T}$ in powers of $\epsilon$ using the solutions of systems of Poisson equations evaluated at the origin. In Section 4 , we give a procedure for evaluating the solutions of the systems of Poisson equations at the origin. Using the techniques developed in the previous sections, in Section 5 we compute expansions of joint moments of $T$ and $X_{T}$ in powers of $\epsilon$ in terms of curvature tensors. These expansions are then used in Section 6 to give precise equivalences between asymptotic Brownian properties and curvature conditions.

Harmonic spaces. Let $r=d(\cdot, m)$ denote the distance function from $m \in M$ and let $S$ denote the unit sphere in the tangent space $T_{m} M$. The manifold $M$ is said to be locally harmonic if, for all $m \in M$, there exists a ball $B_{m}(\epsilon)$ such that $\Delta r^{2}$ is a function depending only on $r$ inside $B_{m}(\epsilon)$. Equivalently, $M$ is locally harmonic if, for all $m \in M$, for all smooth functions $f$ defined on $S$, and for sufficiently small $\epsilon>0$,

$$
E_{m}\left[f\left(\exp _{m}^{-1}\left(X_{T}\right) / \epsilon\right)\right]=\int_{S} f(z) d \sigma(z),
$$

where $d \sigma$ denotes the uniform probability measure on $S$. In particular, if $u$ is harmonic in $B_{m}(\epsilon)$ and $u(x)=f\left(\exp _{m}^{-1}(x) / \epsilon\right)$ on $S_{m}(\epsilon)$ then the expectation on the left in (1.2) equals $u(m)$. Then (1.2) is a mean value property and gives an equivalent definition of locally harmonic spaces. See [RWW], [K].

The class of locally harmonic spaces includes Riemannian manifolds locally isometric to Euclidean space or rank one symmetric spaces [B]. Nonsymmetric examples of harmonic spaces have also been found [DR]. One approach to classifying harmonic spaces is to consider curvature conditions for these spaces.

We fix a point $m \in M$ and represent all tensors with respect to normal coordinates at $m$. No distinction is made between upper and lower indices and we use the summation convention for any repeated indices, unless otherwise noted. Let $R_{i j k l}$ denote the Riemann curvature tensor, $\rho_{i j}=R_{i a j a}$ denote the Ricci curvature tensor, and $\tau=\rho_{a a}$ denote scalar curvature. The metric tensor is denoted $g_{i j}$ and equals the Kronecker delta $\delta_{i j}$ at $m$.

For a locally harmonic manifold $M$, the second order curvature condition,

$$
\left(\rho_{i j}\right)_{m}=(1 / n)\left(\tau g_{i j}\right)_{m}
$$

holds for each $m \in M$ and $\tau_{m}$ is constant on $M$; i.e.,

$$
\left(\nabla_{i} \tau\right)_{m}=0
$$

holds for each $m \in M$. A manifold with these properties is said to be an Einstein manifold. If (1.3) holds for each $m \in M$ and $n>2$, then the scalar curvature is constant by Schur's theorem.

Let $\sum_{P(i j k l)}$ denote summation over all permutations of the indices $i, j, k$, and $l$. At the next level of curvature conditions for locally harmonic manifold $M$, the fourth order condition,

$$
\sum_{P(i j k l)}\left(R_{i p j q} R_{k p l q}\right)_{m}=H_{m} \sum_{P(i j k l)}\left(g_{i j} g_{k l}\right)_{m},
$$


holds for each $m \in M$ and

$$
H_{m}=\frac{\left(2\|\rho\|^{2}+3\|R\|^{2}\right)_{m}}{2 n(n+2)}
$$

is constant on $M$. A consequence of the curvature conditions (1.3) and (1.5) is the super-Einstein condition,

$$
\left(R_{i p q r} R_{j p q r}\right)_{m}=(1 / n)\left(\|R\|^{2} g_{i j}\right)_{m} .
$$

If $M$ is an Einstein manifold, (1.7) holds for each $m \in M$, and $n>4$, then $\|R\|^{2}$ and $H_{m}$ are constant by a Schur type theorem [B; p. 165]. Information about curvature conditions for harmonic spaces can be found in [RWW] or [B].

Statement of results. Consider the asymptotic mean value condition (AMVC):

$$
E_{m}\left[f\left(\exp _{m}^{-1}\left(X_{T}\right) / \epsilon\right)\right]=\int_{S} f(z) d \sigma(z)+o\left(\epsilon^{k}\right), \quad \epsilon \downarrow 0,
$$

for every $f$, a smooth function on $S$, and for all $m \in M$. It is shown in [L1] that if AMVC holds with $k=2$, then the second order curvature condition (1.3) holds on $M$. We shall also show that the converse is true. If $n=2$ and AMVC holds with $k=3$ then $M$ has constant scalar curvature, and hence constant curvature [P2]. This problem was also considered in [KO1] using a different approach. We also show, conversely, that constant scalar curvature and (1.3) imply AMVC with $k=3$.

We extend the results of [GP], [L1], and [P2] to give an asymptotic expansion of the left side of (1.8) up to order $\epsilon^{5}, \epsilon \downarrow 0$. We then use this to show that curvature conditions (1.3), (1.4), and (1.5) hold on $M$ if and only if AMVC holds with $k=4$. Furthermore, AMVC holds with $k=5$ if and only if, in addition, $H_{m}$ is constant on $M$. This also is an improvement of the results of [KO1].

For $M$ a locally harmonic space, Brownian motion also has the property that $X_{T}$ and $T$ are independent random variables if $\epsilon>0$ is sufficiently small [KO2], [L2]. This property is weaker than harmonicity. For example the metric product of $S^{3}$ and $H^{3}$, the three-dimensional spaces with constant curvature 1 and -1 , respectively, has the independence property but is not an Einstein manifold and hence not harmonic [Hu].

Weaker curvature conditions have been found for manifolds with this independence property. Consider the asymptotic uncorrelated condition (AUC):

$$
E_{m}\left[T f\left(\exp _{m}^{-1}\left(X_{T}\right) / \epsilon\right)\right]-E_{m}[T] E_{m}\left[f\left(\exp _{m}^{-1}\left(X_{T}\right) / \epsilon\right)\right]=o\left(\epsilon^{k+2}\right), \quad \epsilon \downarrow 0,
$$

for every $f$, a smooth function on $S$, and for all $m \in M$. It has been shown that if AUC holds with $k=3$ then $M$ has constant scalar curvature [L1]. In [KO2] it is shown that if $T$ and $X_{T}$ are independent for all normal balls at each $m \in M$ then $M$ has constant scalar curvature,

$\left(3 \nabla_{p} \nabla_{p} \rho_{i j}-4 \rho_{i p} \rho_{j p}+2 R_{i p j q} \rho_{p q}+2 R_{i p q r} R_{j p q r}\right)_{m}=(1 / n)\left[\left(2\|R\|^{2}-2\|\rho\|^{2}\right) g_{i j}\right]_{m}$ holds for every $m \in M$ and $\|R\|^{2}-\|\rho\|^{2}$ is constant on $M$. If $M$ is an Einstein manifold, then the super-Einstein condition follows from (1.10).

We extend the method of [GP] and [L1] and give an asymptotic expansion of the left side of (1.9) up to order $\epsilon^{7}, \epsilon \downarrow 0$, and show that we actually have 
equivalences between the curvature conditions above and AUC for appropriate values of $k$. We show that AUC holds with $k=3$ if and only if $M$ has constant scalar curvature, AUC holds with $k=4$ if and only if $M$ has constant scalar curvature and (1.10) holds on $M$, and AUC holds with $k=5$ if and only if scalar curvature and $\|R\|^{2}-\|\rho\|^{2}$ are constant and (1.10) holds on $M$.

\section{WORKING ON THE UNIT BALL}

We will translate the problem from the geodesic ball about $m \in M$ to the unit ball in $T_{m} M$, the tangent space at $m$. We consider values of $\epsilon$ less than $\epsilon_{1}$ where $\epsilon_{1}$ is chosen such that the exponential map is non-singular in $B_{0}\left(\epsilon_{1}\right)$, the ball of radius $\epsilon_{1}$ in $T_{m} M$. Let $B$ and $S$ denote the unit ball and unit sphere, respectively, in $T_{m} M$. We define $\Phi_{\epsilon}: C^{\infty}(\bar{B}) \rightarrow C^{\infty}\left(\overline{B_{m}(\epsilon)}\right)$ by

$$
\left(\Phi_{\epsilon} f\right)\left(\exp _{m} x\right) \doteq f(x / \epsilon) \text {. }
$$

Working in the unit ball, we find it convenient to use homogeneous harmonic polynomials defined as follows. For $x, z \in \mathbb{R}^{n}$, let

$$
K(x, z) \doteq \frac{|z|^{2}-|x|^{2}}{|x-z|^{n}} .
$$

Any $k$ th derivative of $K$,

$$
F(z)=\left.\frac{\partial^{k}}{\partial x_{i_{k}} \ldots \partial x_{i_{1}}} K(x, z)\right|_{x=0}
$$

(where repetitions among the indices $i_{1}, i_{2}, \ldots, i_{k}$ are possible) is harmonic and homogeneous of degree $2-k-n$ and therefore, the Kelvin transform, $|z|^{n+2 k-2} F(z)$, is harmonic and homogeneous of degree $k$. We define the homogeneous harmonic polynomial $Q_{i_{1} \ldots i_{k}}$ with coefficients $Q_{i_{1} \ldots i_{k}}^{a_{1} \ldots a_{k}}$ as follows:

$$
\begin{aligned}
Q_{i_{1} \ldots i_{k}}(z) & =\sum_{a_{1}, \ldots, a_{k}=1}^{n} Q_{i_{1} \ldots i_{k}}^{a_{1} \ldots a_{k}} z_{a_{1}} \cdots z_{a_{k}} \\
& \left.\doteq \frac{|z|^{n+2 k-2}}{n(n+2) \cdots(n+2 k-2)} \cdot \frac{\partial^{k}}{\partial x_{i_{k}} \ldots \partial x_{i_{1}}} K(x, z)\right|_{x=0}
\end{aligned}
$$

In particular, we take the version of the coefficients $Q_{i_{1} \ldots i_{k}}^{a_{1} \ldots a_{k}}$ symmetrized with respect to $a_{1}, \ldots, a_{k}$.

By induction, one can show that partial derivatives of $K$ with respect to $x$ have the form

$$
\begin{aligned}
& \frac{\partial^{k}}{\partial x_{i_{k}} \ldots \partial x_{i_{1}}} K(x, z) \\
& =\frac{n(n+2) \cdots(n+2 k-2)\left(z_{i_{1}}-x_{i_{1}}\right) \cdots\left(z_{i_{k}}-x_{i_{k}}\right)\left(|z|^{2}-|x|^{2}\right)}{|x-z|^{n+2 k}} \\
& \quad+\frac{|z|^{2} p(x, z)}{|x-z|^{n+2 k-2}}+\frac{|x|^{2} q(x, z)}{|x-z|^{n+2 k-2}}+\sum_{j=1}^{k} \frac{x_{i_{j}} r_{j}(x, z)}{|x-z|^{n+2 k-2}}
\end{aligned}
$$

where $p, q$, and $r_{j}, j=1, \ldots, k$, are polynomials in $x$ and $z$ and depend on $i_{1}, \ldots, i_{k}$. It follows that

$$
Q_{i_{1} \ldots i_{k}}(z)=z_{i_{1}} \cdots z_{i_{k}}+|z|^{2} \hat{Q}_{i_{1} \ldots i_{k}}(z)
$$


where $\hat{Q}_{i_{1} \ldots i_{k}}$ is a homogeneous polynomial of degree $k-2$. Since $Q_{i_{1} \ldots i_{k}}$ is harmonic and homogeneous of degree $k$, it is the harmonic projection of the monomial $z_{i_{1}} \cdots z_{i_{k}}$ in the space of homogeneous polynomials of degree $k$. Furthermore, the polynomials $Q_{i_{1} \ldots i_{k}}(z)$ span the space of harmonic homogeneous polynomials of degree $k$. Since the restrictions of harmonic homogeneous polynomials to $S$ form a dense set in the space of continuous functions on $S$, we need only consider functions $f$ from this class in conditions (1.8) and (1.10). See [VK, Section 9.2.3].

Let $\sum_{P\left(i_{1} \ldots i_{k}\right)}$ denote summation over the permutations of the indices $i_{1}, \ldots$, $i_{k}$. Then we have from [VK, ibid.] that $Q_{i_{1} \ldots i_{k}}(z)$ equals

$$
\begin{aligned}
& z_{i_{1}} \cdots z_{i_{k}} \\
& +\sum_{j=1}^{[k / 2]} \sum_{P\left(i_{1} \ldots i_{k}\right)} \frac{(-1)^{j}|z|^{2 j} \delta_{i_{1} i_{2}} \cdots \delta_{i_{2 j-1} i_{2 j}} z_{i_{2 j+1}} \cdots z_{i_{k}}}{2^{j} j !(k-2 j) !(n+2 k-4)(n+2 k-6) \cdots(n+2 k-2 j-2)} .
\end{aligned}
$$

The coefficient $Q_{i_{1} \ldots i_{k}}^{a_{1} \ldots a_{k}}$ equals

$$
\begin{aligned}
& \frac{1}{k !} \sum_{P\left(a_{1} \ldots a_{k}\right)}\left\{\delta_{i_{1} a_{1}} \cdots \delta_{i_{k} a_{k}}\right. \\
& \left.\quad+\sum_{j=1}^{[k / 2]} \sum_{P\left(i_{1} \ldots i_{k}\right)} \frac{(-1)^{j} \delta_{i_{1} i_{2}} \cdots \delta_{i_{2 j-1} i_{2 j}} \delta_{a_{1} a_{2}} \cdots \delta_{a_{2 j-1} a_{2 j}} \delta_{i_{2 j+1} a_{2 j+1}} \cdots \delta_{i_{k} a_{k}}}{2^{j} j !(k-2 j) !(n+2 k-4)(n+2 k-6) \cdots(n+2 k-2 j-2)}\right\} .
\end{aligned}
$$

In particular, we have

$$
\begin{aligned}
Q_{i}(z) & =z_{i} \\
Q_{i j}(z) & =z_{i} z_{j}-\frac{1}{n} \delta_{i j}|z|^{2}, \\
Q_{i j k}(z)= & z_{i} z_{j} z_{k}-\frac{1}{2(n+2)} \sum_{P(i j k)} \delta_{i j} z_{k}|z|^{2}, \\
Q_{i j k l}(z)= & z_{i} z_{j} z_{k} z_{l}-\frac{1}{4(n+4)} \sum_{P(i j k l)} \delta_{i j} z_{k} z_{l}|z|^{2} \\
& +\frac{1}{8(n+2)(n+4)} \sum_{P(i j k l)} \delta_{i j} \delta_{k l}|z|^{4}, \\
Q_{i j k l h}(z)= & z_{i} z_{j} z_{k} z_{l} z_{h}-\frac{1}{12(n+6)} \sum_{P(i j k l h)} \delta_{i j} z_{k} z_{l} z_{h}|z|^{2} \\
& +\frac{1}{8(n+4)(n+6)} \sum_{P(i j k l h)} \delta_{i j} \delta_{k l} z_{h}|z|^{4} .
\end{aligned}
$$

\section{ASYMPTOTIC EXPANSIONS FROM PDE'S}

We use approximate solutions of partial differential equations to compute asymptotic expansions of $E_{m}\left[T^{l}\left(\Phi_{\epsilon} f\right)\left(X_{T}\right)\right]$ in $\epsilon$. Then we will find the approximate solutions as solutions of systems of Poisson equations with boundary 
conditions. We only consider the cases $l=0,1$ but this method can be extended for any positive integer $l$.

Proposition 3.1. Let $A=\frac{1}{2} \Delta$. Let $f$ be a smooth function on $S$. Suppose $\hat{u}_{\epsilon}$ and $\hat{v}_{\epsilon}$ satisfy the following as $\epsilon \downarrow 0$ :

$$
\begin{aligned}
\Phi_{\epsilon}^{-1} A \Phi_{\epsilon} \hat{u}_{\epsilon}=O\left(\epsilon^{k-1}\right) \text { in } B, & \hat{u}_{\epsilon}=f \text { on } S, \\
\Phi_{\epsilon}^{-1} A \Phi_{\epsilon} \hat{v}_{\epsilon}+\hat{u}_{\epsilon}=O\left(\epsilon^{k+1}\right) \text { in } B, & \hat{v}_{\epsilon}=0 \text { on } S .
\end{aligned}
$$

Then

$$
\begin{aligned}
E_{m}\left[\left(\Phi_{\epsilon} f\right)\left(X_{T}\right)\right] & =\hat{u}_{\epsilon}(0)+O\left(\epsilon^{k+1}\right), \\
E_{m}\left[T\left(\Phi_{\epsilon} f\right)\left(X_{T}\right)\right] & =\hat{v}_{\epsilon}(0)+O\left(\epsilon^{k+3}\right) .
\end{aligned}
$$

Set $f \equiv 1$ and $\hat{u}_{\epsilon} \equiv 1$, then Proposition 3.1 implies:

Corollary 3.2. Suppose $\hat{v}_{\epsilon}$ satisfies the following as $\epsilon \downarrow 0$ :

$$
\Phi_{\epsilon}^{-1} A \Phi_{\epsilon} \hat{v}_{\epsilon}+1=O\left(\epsilon^{k+1}\right) \text { in } B, \quad \hat{v}_{\epsilon}=0 \text { on } S .
$$

Then

$$
E_{m}[T]=\hat{v}_{\epsilon}(0)+O\left(\epsilon^{k+3}\right) .
$$

Perturbation method. Before proceeding with the proof of Proposition 3.1, we describe how solutions of (3.1) can be found using systems of Poisson equations. The operator $A=\frac{1}{2} \Delta$ has the following homogeneous decomposition with respect to normal coordinates $\left(x^{j}\right)$ [GP]:

$$
A=\frac{1}{2} \Delta_{-2}+\frac{1}{2} \sum_{i=0}^{\infty} \Delta_{i} \quad\left(\Delta_{-1}=0\right)
$$

Here $\Delta_{-2}$ is the ordinary Euclidean Laplacian and $\Delta_{i}$ is a second order linear differential operator with polynomial coefficients of the form $\Delta_{i}=q_{i}^{j} \partial_{j}+q_{i}^{j k} \partial_{j} \partial_{k}$ where $q_{i}^{j}$ and $q_{i}^{j k}$ are respectively $(i+1)$-degree and $(i+2)$-degree homogeneous polynomials in $\left(x^{j}\right) . \Delta_{i}$ maps $k$-degree homogeneous polynomials to $(k+i)$-degree homogeneous polynomials.

Proposition 3.3. Let $f$ be a smooth function on $S$. Suppose that $u_{j}$ and $v_{j}$, $0 \leq j \leq k$, satisfy

$$
\begin{aligned}
\Delta_{-2} u_{0} & =0 \text { in } B, \quad u_{0}=f \text { on } S, \\
\Delta_{-2} u_{j}+\sum_{i=2}^{j} \Delta_{i-2} u_{j-i} & =0 \text { in } B, \quad u_{j}=0 \text { on } S \quad(1 \leq j \leq k), \\
\Delta_{-2} v_{j}+\sum_{i=2}^{j} \Delta_{i-2} v_{j-i}+2 u_{j} & =0 \text { in } B, \quad v_{j}=0 \text { on } S \quad(0 \leq j \leq k) .
\end{aligned}
$$

Let $\hat{u}_{\epsilon}$ and $\hat{v}_{\epsilon}$ be defined as follows:

$$
\hat{u}_{\epsilon}=\sum_{j=0}^{k} \epsilon^{j} u_{j}, \quad \hat{v}_{\epsilon}=\sum_{j=0}^{k} \epsilon^{j+2} v_{j} .
$$

Then $\hat{u}_{\epsilon}$ and $\hat{v}_{\epsilon}$ are solutions of (3.1). 
Proof. On $S, \hat{u}_{\epsilon}=u_{0}+0=f$ and $\hat{v}_{\epsilon}=0$. In $B$, the homogeneous decomposition is

$$
\Phi_{\epsilon}^{-1} A \Phi_{\epsilon}=\frac{1}{2} \epsilon^{-2} \Delta_{-2}+\frac{1}{2} \sum_{i=0}^{\infty} \epsilon^{i} \Delta_{i},
$$

where normal coordinates are identified with coordinates in the tangent space. Therefore we have, using (3.6) and (3.7),

$$
\begin{aligned}
\boldsymbol{\Phi}_{\epsilon}^{-1} A \boldsymbol{\Phi}_{\epsilon} \hat{u}_{\epsilon}= & \frac{1}{2} \sum_{j=0}^{k} \epsilon^{j-2}\left[\Delta_{-2} u_{j}+\sum_{i=2}^{j} \Delta_{i-2} u_{j-i}\right] \\
& +\frac{1}{2} \sum_{j=0}^{k} \epsilon^{k-1}\left[\sum_{i=k-j+1}^{\infty} \epsilon^{i+j-k-1} \Delta_{i-2} u_{j}\right] \\
= & \frac{1}{2} \sum_{j=0}^{k} \epsilon^{k-1}\left(\tilde{\Delta}_{k-j-1} u_{j}\right),
\end{aligned}
$$

where $\tilde{\Delta}_{k-j-1}$ is a second order linear differential operator with bounded coefficients.

By repeated application of Schauder's estimate, $\left\|u_{j}\right\|_{2} \leq K_{j}\|f\|_{2}$, where the constant $K_{j}$ does not depend on $f$. Therefore, $\left|\Phi_{\epsilon}^{-1} A \Phi_{\epsilon} \hat{u}_{\epsilon}\right| \leq K \epsilon^{k-1}\|f\|_{2}=$ $O\left(\epsilon^{k-1}\right)$.

Similarly, using repeated applications of Schauder's estimate, we have

$$
\Phi_{\epsilon}^{-1} A \Phi_{\epsilon} \hat{v}_{\epsilon}+\hat{u}_{\epsilon}=\frac{1}{2} \sum_{j=0}^{k} \epsilon^{k+1}\left(\tilde{\Delta}_{k-j-1} v_{j}\right)=O\left(\epsilon^{k+1}\right) .
$$

Proof of Proposition 3.1. We first prove the following lemma using the perturbation method.

Lemma 3.4. $E_{m}[T]=O\left(\epsilon^{2}\right)$ and $E_{m}\left[T^{2}\right]=O\left(\epsilon^{4}\right)$.

Proof. Let $v$ and $w$ be solutions of

$$
\begin{aligned}
\Delta_{-2} v+2 & =0 \text { in } B, \quad v=0 \text { on } S, \\
\Delta_{-2} w+2 v & =0 \text { in } B, \quad w=0 \text { on } S,
\end{aligned}
$$

Let $\tilde{v}_{\epsilon}=\Phi_{\epsilon}\left(\epsilon^{2} v\right)$ and $\tilde{w}_{\epsilon}=\Phi_{\epsilon}\left(\epsilon^{4} w\right)$. Then by Proposition 3.3,

$$
\begin{aligned}
A \tilde{v}_{\epsilon}+1 & =O(\epsilon) \text { in } B_{m}(\epsilon), & \tilde{v}_{\epsilon} & =0 \text { on } S_{m}(\epsilon), \\
A \tilde{w}_{\epsilon}+\tilde{v}_{\epsilon} & =O\left(\epsilon^{3}\right) \text { in } B_{m}(\epsilon), & \tilde{w}_{\epsilon} & =0 \text { on } S_{m}(\epsilon) .
\end{aligned}
$$

Therefore by Dynkin's formula we have

$$
\begin{aligned}
0=E_{m}\left[\tilde{v}_{\epsilon}\left(X_{T}\right)\right] & =\tilde{v}_{\epsilon}(m)+E_{m}\left[\int_{0}^{T}\left(A \tilde{v}_{\epsilon}\right)\left(X_{s}\right) d s\right] \\
& =\epsilon^{2} v(0)-E_{m}\left[\int_{0}^{T}(1+O(\epsilon)) d s\right] \\
& =\epsilon^{2} v(0)-(1+O(\epsilon)) E_{m}[T] .
\end{aligned}
$$


Thus $E_{m}[T]=O\left(\epsilon^{2}\right)$. Also by Dynkin's formula,

$$
\begin{aligned}
0=E_{m}\left[\tilde{w}_{\epsilon}\left(X_{T}\right)\right] & =\tilde{w}_{\epsilon}(m)+E_{m}\left[\int_{0}^{T}\left(A \tilde{w}_{\epsilon}\right)\left(X_{s}\right) d s\right] \\
& =\tilde{w}_{\epsilon}(m)-E_{m}\left[\int_{0}^{T}\left\{\tilde{v}_{\epsilon}\left(X_{s}\right)+O\left(\epsilon^{3}\right)\right\} d s\right] \\
& =\tilde{w}_{\epsilon}(m)-E_{m}\left[\int_{0}^{T} \tilde{v}_{\epsilon}\left(X_{s}\right) d s\right]+O\left(\epsilon^{5}\right) .
\end{aligned}
$$

By Itô's formula and (3.14) we have

$$
\begin{aligned}
0=E_{m}\left[T \tilde{v}_{\epsilon}\left(X_{T}\right)\right] & =0+E_{m}\left[\int_{0}^{T}\left\{s\left(A \tilde{v}_{\epsilon}\right)\left(X_{s}\right)+\tilde{v}_{\epsilon}\left(X_{s}\right)\right\} d s\right] \\
& =E_{m}\left[\int_{0}^{T}\left\{-s(1+O(\epsilon))+\tilde{v}_{\epsilon}\left(X_{s}\right)\right\} d s\right] \\
& =-(1+O(\epsilon)) E_{m}\left[T^{2}\right] / 2+\tilde{w}_{\epsilon}(m)+O\left(\epsilon^{5}\right) \\
& =-(1+O(\epsilon)) E_{m}\left[T^{2}\right] / 2+\epsilon^{4} w(0)+O\left(\epsilon^{5}\right) .
\end{aligned}
$$

Thus $E_{m}\left[T^{2}\right]=O\left(\epsilon^{4}\right)$.

Proof of Proposition 3.1. Let $\tilde{u}_{\epsilon}$ and $\tilde{v}_{\epsilon}$ be defined as follows:

$$
\tilde{u}_{\epsilon}=\Phi_{\epsilon} \hat{u}_{\epsilon}, \quad \tilde{v}_{\epsilon}=\Phi_{\epsilon} \hat{v}_{\epsilon},
$$

where $\hat{u}_{\epsilon}$ and $\hat{v}_{\epsilon}$ are defined as in (3.7). Then we have

$$
\begin{aligned}
A \tilde{u}_{\epsilon} & =O\left(\epsilon^{k-1}\right) \text { in } B_{m}(\epsilon), & \tilde{u}_{\epsilon}=\Phi_{\epsilon} f \text { on } S_{m}(\epsilon), \\
A \tilde{v}_{\epsilon}+\tilde{u}_{\epsilon} & =O\left(\epsilon^{k+1}\right) \text { in } B_{m}(\epsilon), & \tilde{v}_{\epsilon}=0 \text { on } S_{m}(\epsilon) .
\end{aligned}
$$

By Dynkin's formula,

$$
\begin{aligned}
E_{m}\left[\left(\Phi_{\epsilon} f\right)\left(X_{T}\right)\right] & =E_{m}\left[\tilde{u}_{\epsilon}\left(X_{T}\right)\right] \\
& =\tilde{u}_{\epsilon}(m)+E_{m}\left[\int_{0}^{T}\left(A \tilde{u}_{\epsilon}\right)\left(X_{s}\right) d s\right] \\
& =\hat{u}_{\epsilon}(0)+E_{m}\left[\int_{0}^{T} O\left(\epsilon^{k-1}\right) d s\right] \\
& =\hat{u}_{\epsilon}(0)+O\left(\epsilon^{k+1}\right) .
\end{aligned}
$$

By Itô's formula and Dynkin's formula,

$$
\begin{aligned}
E_{m}\left[T\left(\Phi_{\epsilon} f\right)\left(X_{T}\right)\right] & =E_{m}\left[T \tilde{u}_{\epsilon}\left(X_{T}\right)\right] \\
& =0+E_{m}\left[\int_{0}^{T}\left\{s\left(A \tilde{u}_{\epsilon}\right)\left(X_{s}\right)+\tilde{u}_{\epsilon}\left(X_{s}\right)\right\} d s\right] \\
& =E_{m}\left[\int_{0}^{T}\left\{\tilde{u}_{\epsilon}\left(X_{s}\right)+s O\left(\epsilon^{k-1}\right)\right\} d s\right] \\
& =E_{m}\left[\int_{0}^{T} \tilde{u}_{\epsilon}\left(X_{s}\right) d s\right]+O\left(\epsilon^{k+3}\right) \\
& =\hat{v}_{\epsilon}(0)+O\left(\epsilon^{k+3}\right) .
\end{aligned}
$$




\section{Evaluating solutions at 0}

Pizetti formulas. In the previous section, we reduced the problem to a system of Poisson equations with boundary conditions. We derive tools to evaluate solutions of these equations at zero. We give Pizetti's formula in $\mathbb{R}^{n}[\mathrm{CH}, \mathrm{pp}$. 287-289]:

Lemma 4.1. Let $\sigma_{r}$ denote the uniform probability measure on $S_{0}(r)$. Suppose $\Delta_{-2}^{m+1} u=0$, then

$$
\int_{S_{0}(r)} u d \sigma_{r}=u(0)+\sum_{h=1}^{m} \frac{1}{2^{h} h ! n(n+2) \cdots(n+2 h-2)}\left(\Delta_{-2}^{h} u\right)(0) r^{2 h} .
$$

We define the following dimension constants for $k \geq 0$ :

$$
\phi_{h}^{k} \doteq \begin{cases}1, & h=0, \\ \frac{1}{2^{h} h !(n+2 k)(n+2 k+2) \cdots(n+2 k+2 h-2)}, & h>0 .\end{cases}
$$

As shown in [L1], Lemma 4.1 implies

Corollary 4.2. Suppose $\Delta_{-2}^{m+1} u=0$ in $B$ and $u=0$ on $S$, then

$$
u(0)=-\sum_{h=1}^{m} \phi_{h}^{0}\left(\Delta_{-2}^{h} u\right)(0)
$$

We extend this formula to derivatives of $u$. Let

$$
Q_{i_{1} \ldots i_{k}}(\nabla) \doteq \sum_{j_{1}, \ldots, j_{k}=1}^{n} Q_{i_{1} \ldots i_{k}}^{j_{1} \ldots j_{k}} \partial_{j_{1}} \ldots \partial_{j_{k}} .
$$

We have then:

Lemma 4.3. Suppose $\Delta_{-2}^{m+1} u=0$ in $B$ and $u=0$ on $S$, then

$$
\left[Q_{i_{1} \ldots i_{k}}(\nabla) u\right](0)=-\sum_{h=1}^{m} \phi_{h}^{k}\left[Q_{i_{1} \ldots i_{k}}(\nabla) \Delta_{-2}^{h} u\right](0) .
$$

Proof. For simplicity we write $Q$ for $Q_{i_{1} \ldots i_{k}}$. Let $U(x)=Q(x) u(x)$. Since $Q$ and its derivatives are harmonic, by repeated application of the product rule for the Laplacian, we have the following formula for $\Delta_{-2}^{h} U$ :

$$
\Delta_{-2}^{h} U=\sum_{j=0}^{h}\left(\begin{array}{l}
h \\
j
\end{array}\right) 2^{j} \sum_{a_{1}, \ldots, a_{j}=1}^{n}\left(\partial_{a_{1}} \ldots \partial_{a_{j}} Q\right)\left(\partial_{a_{1}} \ldots \partial_{a_{j}} \Delta_{-2}^{h-j} u\right) .
$$

Since $Q$ is a homogeneous polynomial of degree $k$,

$$
\begin{aligned}
\left(\partial_{a_{1}} \ldots \partial_{a_{j}} Q\right)(0) & =0, & & j<k, \\
\partial_{a_{1}} \ldots \partial_{a_{j}} Q & =k ! Q_{i_{1} \ldots i_{k}}^{a_{1} \ldots a_{k}}, & & j=k, \\
\partial_{a_{1}} \ldots \partial_{a_{j}} Q & =0, & & j>k .
\end{aligned}
$$

Therefore,

$$
\left(\Delta_{-2}^{h} U\right)(0)= \begin{cases}\frac{h ! 2^{k}}{(h-k) !}\left[Q(\nabla) \Delta_{-2}^{h-k} u\right](0), & h \geq k, \\ 0, & h<k .\end{cases}
$$


By (4.6) and (4.7), $\Delta_{-2}^{m+k+1} U=0$ in $B$. Also $U=0$ on $S . U(0)=0$ since $Q(0)=0$. Therefore by Corollary 4.2 ,

$$
0=-\sum_{h=k}^{m+k} \frac{h ! 2^{k}}{(h-k) !} \phi_{h}^{0}\left[Q(\nabla) \Delta_{-2}^{h-k} u\right](0) .
$$

Equation (4.5) follows by replacing the $h$ with $h+k$ and solving for $[Q(\nabla) u](0)$.

In particular, we can now compute derivatives of $u$ at 0 in terms of derivatives of $\Delta_{-2} u$ at 0 .

Corollary 4.4. Suppose $\Delta_{-2}^{m+1} u=0$ in $B$ and $u=0$ on $S$. Then

$$
\begin{aligned}
& \left(\partial_{i_{1}} \cdots \partial_{i_{k}} u\right)(0)=-\sum_{h=1}^{m} \phi_{h}^{k}\left(\partial_{i_{1}} \cdots \partial_{i_{k}} \Delta_{-2}^{h} u\right)(0) \\
& \quad-\sum_{j=1}^{[k / 2]} \sum_{h=0}^{m-j} \sum_{P\left(i_{1} \ldots i_{k}\right)} \frac{\phi_{h}^{k}(-1)^{j} \delta_{i_{1} i_{2}} \cdots \delta_{i_{2 j-1} i_{2 j}}\left(\partial_{i_{2 j+1}} \cdots \partial_{i_{k}} \Delta_{-2}^{h+j} u\right)(0)}{2^{j} j !(k-2 j) !(n+2 k-4)(n+2 k-6) \cdots(n+2 k-2 j-2)} .
\end{aligned}
$$

Reduction process. The functions $u_{j}(1 \leq j \leq k)$ and $v_{j}(0 \leq j \leq k)$ defined by (3.6) above are solutions of Poisson equations of the form

$$
\Delta_{-2} V_{j}+\sum_{i=2}^{j} \Delta_{i-2} V_{j-i}+2 U_{j}=0 \text { in } B,
$$

with boundary condition $V_{j}=0$ on $S$. Here $\Delta_{-2} V_{j}$ can be expressed in terms of $U_{j}$ and $V_{i}, i<j$. If there exists $m$ such that $\Delta_{-2}^{m+1} V=0$ then Corollaries 4.2 and 4.4 show how to express $V_{j}$ and its derivatives evaluated at 0 in terms of $U_{j}$ and $V_{i}, i<j$, and their derivatives evaluated at 0 . We develop formulas to assist in the reduction process and to verify that the hypothesis of Corollaries 4.2 and 4.4 is satisfied by the solutions of (3.6).

Define the differential operator $A_{k}^{j}$ as follows:

$$
A_{k}^{j} \doteq \begin{cases}\Delta_{k}, & j=0, \\ \Delta_{-2} A_{k}^{j-1}-A_{k}^{j-1} \Delta_{-2}, & j \geq 1 .\end{cases}
$$

It follows easily by induction that each $A_{k}^{j}$ has the following properties:

Lemma 4.5. $A_{k}^{j}$ is a linear differential operator, of order $2+j$, with polynomial coefficients of degree $\leq 2+k-j$ and $\geq 1+k-2 j$ (where a polynomial of negative degree is understood to be the 0 polynomial). Furthermore,

$$
\Delta_{-2}^{m} \Delta_{k}=\sum_{j=0}^{m}\left(\begin{array}{c}
m \\
j
\end{array}\right) A_{k}^{j} \Delta_{-2}^{m-j} .
$$

We now check the hypothesis of Corollaries 4.2 and 4.4 . 
Lemma 4.6. Suppose for $0 \leq j \leq k, u_{j}$ and $v_{j}$ satisfy (3.6). Then $\Delta_{-2}^{2 k+1} u_{k}=0$ and $\Delta_{-2}^{2 k+2} v_{k}=0$ in $B$.

Proof. We proceed by induction. First consider $u_{j}, 0 \leq j \leq k$. By (3.6), $\Delta_{-2} u_{0}=0$ in $B$. Suppose $\Delta_{-2}^{2 j+1} u_{j}=0$ in $B, j<k$. Then by (3.6) and Lemma 4.5,

$$
\Delta_{-2}^{2 k+1} u_{k}=-\sum_{i=2}^{k} \sum_{h=0}^{2 k}\left(\begin{array}{c}
2 k \\
h
\end{array}\right) A_{i-2}^{h} \Delta_{-2}^{2 k-h} u_{k-i} .
$$

Now $\Delta_{-2}^{2 k-h} u_{k-i}=0$ for $i \geq 1$ and $h \leq i$. By Lemma 4.5, $A_{i-2}^{h}=0$ for $h>i$. Thus $\Delta_{-2}^{2 k+1} u_{k}=0$ in $B$. Then,

$$
\Delta_{-2}^{2 k+2} v_{k}=-\sum_{i=2}^{k} \sum_{h=0}^{2 k+1}\left(\begin{array}{c}
2 k+1 \\
h
\end{array}\right) A_{i-2}^{h} \Delta_{-2}^{2 k+1-h} v_{k-i}-2 \Delta_{-2}^{2 k+1} u_{k}=0
$$

in $B$.

Lemma 4.6 implies that we may apply Corollaries 4.2 and 4.4 to the solutions of (3.6) and thus we may ultimately express each of $u_{i}(0)$ and $v_{i}(0)$ in terms of $u_{0}$ and its derivatives evaluated at 0 . In Section 5, we give the results of this reduction process for $0 \leq i \leq 4$. Before we do this, however, we give formulas for computing $u_{0}$ and its derivatives at 0 .

Derivatives of $u_{0}$ at 0 . Let $u_{0}$ denote the solution of

$$
\Delta_{-2} u_{0}=0 \text { in } B, \quad u_{0}=f \text { on } S
$$

as before. Then by the Poisson formula,

$$
\left(\partial_{i_{1}} \partial_{i_{2}} \ldots \partial_{i_{k}} u_{0}\right)(0)=\int_{S} n(n+2) \cdots(n+2 k-2) Q_{i_{1} i_{2} \ldots i_{k}}(z) f(z) d \sigma(z)
$$

where $\sigma$ is the uniform probability measure on $S$.

When a harmonic homogeneous polynomial $Q_{p_{1} \ldots p_{m}}(z)$ is substituted for $f(z)$, we have an explicit computation for the integral.

Lemma 4.7.

$$
\begin{array}{r}
\int_{S} n(n+2) \cdots(n+2 k-2) Q_{i_{1} \ldots i_{k}}(z) Q_{p_{1} \ldots p_{m}}(z) d \sigma(z) \\
= \begin{cases}k ! Q_{i_{1} \ldots i_{k}}^{p_{1} \ldots p_{k}}, & k=m, \\
0, & k \neq m .\end{cases}
\end{array}
$$

where $Q_{i_{1} \ldots i_{k}}^{p_{1} \ldots p_{k}}$ are the coefficients given in (2.8).

Proof. Since the polynomials $Q$ are harmonic,

$$
\Delta_{-2}^{h}\left(Q_{i_{1} \ldots i_{k}} Q_{p_{1} \ldots p_{m}}\right)=2^{h} \sum_{a_{1}, \ldots, a_{h}=1}^{n}\left(\partial_{a_{1}} \ldots \partial_{a_{h}} Q_{i_{1} \ldots i_{k}}\right)\left(\partial_{a_{1}} \ldots \partial_{a_{h}} Q_{p_{1} \ldots p_{m}}\right)
$$


Since the polynomials $Q$ are homogeneous

$$
\left(\partial_{a_{1}} \ldots \partial_{a_{h}} Q_{i_{1} \ldots i_{k}}\right)(0)= \begin{cases}k ! Q_{i_{1} \ldots i_{k}}^{a_{1} \ldots a_{k}}, & h=k, \\ 0, & h \neq k\end{cases}
$$

Since for harmonic function $Q$,

$$
Q_{p_{1} \ldots p_{k}}(\nabla) Q=\partial_{p_{1}} \ldots \partial_{p_{k}} Q
$$

we have

$$
\begin{aligned}
k ! \sum_{a_{1}, \ldots, a_{k}=1}^{n} Q_{i_{1} \ldots i_{k}}^{a_{1} \ldots a_{k}} Q_{p_{1} \ldots p_{k}}^{a_{1} \ldots a_{k}} & =Q_{p_{1} \ldots p_{k}}(\nabla) Q_{i_{1} \ldots i_{k}} \\
& =\partial_{p_{1}} \ldots \partial_{p_{k}} Q_{i_{1} \ldots i_{k}} \\
& =k ! Q_{i_{1} \ldots i_{k}}^{p_{1} \ldots p_{k}}
\end{aligned}
$$

Therefore (4.18) follows by Pizetti's formula, (4.19), (4.20), and (4.22).

\section{RESULTS OF REDUCTION PROCEDURE}

The operators $A_{i}^{j}$ in terms of curvature. We use the summation convention for repeated indices in the following. For convenience we define the following tensors involving curvature with respect to a normal coordinate system:

$$
\begin{aligned}
& (R \# R)_{i j k l} \doteq R_{i a j b} R_{k a l b}, \quad(R \# \nabla \rho)_{i j k} \doteq R_{i a j b} \nabla_{b} \rho_{k a}, \\
& (\rho * \nabla \rho)_{i j k} \doteq \rho_{i a} \nabla_{a} \rho_{j k}, \quad(R * R)_{i j} \doteq R_{i a b c} R_{j a b c}, \\
& (R \# \rho)_{i j} \doteq R_{i a j b} \rho_{a b}, \quad(\rho * \rho)_{i j} \doteq \rho_{i a} \rho_{j a}, \\
& (\rho \# \nabla \rho)_{i} \doteq \rho_{a b} \nabla_{b} \rho_{i a}, \quad(\rho * \nabla \tau)_{i} \doteq \rho_{i a} \nabla_{a} \tau, \\
& \|R\|^{2} \doteq R_{a b c d} R_{a b c d}, \quad\|\rho\|^{2} \doteq \rho_{a b} \rho_{a b} .
\end{aligned}
$$

We note the following symmetries:

$$
\begin{aligned}
(R \# R)_{i j k l}=(R \# R)_{k l i j}=(R \# R)_{j i l k}, \\
(R * R)_{i j}=(R * R)_{j i}, \\
(R \# \rho)_{i j}=(R \# \rho)_{j i}, \\
(\rho * \rho)_{i j}=(\rho * \rho)_{j i},
\end{aligned}
$$

and the following relations:

$$
\begin{gathered}
(R \# R)_{i a j a}=2(R \# R)_{i a a j}=(R * R)_{i j}, \\
(R \# R)_{i j a a}=(R \# \rho)_{i j}, \\
(R * R)_{a a}=\|R\|^{2}, \\
(R \# \rho)_{a a}=(\rho * \rho)_{a a}=\|\rho\|^{2} .
\end{gathered}
$$


The first few $\Delta_{i}$ have been computed [GP], [KO2]:

$$
\begin{aligned}
\Delta_{0}= & (1 / 3) R_{i p j q}(m) x^{p} x^{q} \partial_{i} \partial_{j}-(2 / 3) \rho_{i p}(m) x^{p} \partial_{i}, \\
\Delta_{1}= & (1 / 6)\left(\nabla_{r} R_{i p j q}\right)(m) x^{p} x^{q} x^{r} \partial_{i} \partial_{j} \\
& +(1 / 12)\left[\nabla_{i} \rho_{p q}-6 \nabla_{q} \rho_{i p}\right](m) x^{p} x^{q} \partial_{i}, \\
\Delta_{2}= & (1 / 60)\left[3 \nabla_{s} \nabla_{r} R_{i p j q}+4 R_{r i s h} R_{p j q h}\right](m) x^{p} x^{q} x^{r} x^{s} \partial_{i} \partial_{j} \\
& +(1 / 180)\left[9 \nabla_{i} \nabla_{r} \rho_{p q}-36 \nabla_{r} \nabla_{q} \rho_{p i}+4 \rho_{r h} R_{h p i q}\right. \\
& \left.-16(R \# R)_{i p q r}\right](m) x^{p} x^{q} x^{r} \partial_{i}, \\
\Delta_{3}= & (1 / 90)\left[\nabla_{t} \nabla_{s} \nabla_{r} R_{i p j q}+3 \nabla_{t}\left(R_{r i s h} R_{p j q h}\right)\right](m) x^{p} x^{q} x^{r} x^{s} x^{t} \partial_{i} \partial_{j} \\
& +(1 / 360)\left[6 \nabla_{i} \nabla_{s} \nabla_{r} \rho_{p q}-20 \nabla_{s} \nabla_{r} \nabla_{q} \rho_{p i}+10 R_{r i s k} \nabla_{k} \rho_{p q}\right. \\
& \left.+3 \nabla_{i}(R \# R)_{p q r s}-20 \nabla_{s}(R \# R)_{i p q r}\right](m) x^{p} x^{q} x^{r} x^{s} \partial_{i} .
\end{aligned}
$$

Using the definitions in (4.12) we compute the following operators:

(5.5)

$$
\begin{aligned}
& A_{0}^{1}=-(2 / 3) \rho_{i j} \partial_{i} \partial_{j}, \\
& A_{0}^{j}=0, \quad j \geq 2, \\
& \left.A_{1}^{1}\right|_{0}=-(1 / 3) \nabla_{i} \tau \partial_{i}, \\
& A_{1}^{2}=-2 \nabla_{k} \rho_{i j} \partial_{i} \partial_{j} \partial_{k}, \\
& A_{1}^{j}=0, \quad j \geq 3 \text {, } \\
& \left.A_{2}^{1}\right|_{0}=0 \text {, } \\
& \left.A_{2}^{2}\right|_{0}=-(2 / 45)\left[27 \nabla_{i} \nabla_{j} \tau+9 \nabla_{p} \nabla_{p} \rho_{i j}+38(\rho * \rho)_{i j}\right. \\
& \left.-34(R \# \rho)_{i j}+6(R * R)_{i j}\right] \partial_{i} \partial_{j}, \\
& A_{2}^{3}=-(8 / 15)\left[9 \nabla_{l} \nabla_{k} \rho_{i j}+2(R \# R)_{i j k l}\right] \partial_{i} \partial_{j} \partial_{k} \partial_{l} \text {, } \\
& A_{2}^{j}=0, \quad j \geq 4, \\
& \left.A_{3}^{1}\right|_{0}=0 \text {, } \\
& \left.A_{3}^{2}\right|_{0}=-(1 / 45)\left[18 \nabla_{i} \Delta \tau+40(\rho * \nabla \tau)_{i}-13 \nabla_{i}\left(\|\rho\|^{2}\right)\right. \\
& \left.+60(\rho \# \nabla \rho)_{i}+3 \nabla_{i}\left(\|R\|^{2}\right)\right] \partial_{i}, \\
& \left.A_{3}^{3}\right|_{0}=-(4 / 15)\left[12 \nabla_{k} \nabla_{j} \nabla_{i} \tau+9 \nabla_{k} \nabla_{p} \nabla_{p} \rho_{i j}\right. \\
& +33 \nabla_{k}(\rho * \rho)_{i j}-5(\rho * \nabla \rho)_{i j k} \\
& \left.-19 \nabla_{k}(R \# \rho)_{i j}-50(R \# \nabla \rho)_{i j k}+6 \nabla_{k}(R * R)_{i j}\right] \partial_{i} \partial_{j} \partial_{k} \text {, } \\
& A_{3}^{4}=-(16 / 3)\left[2 \nabla_{h} \nabla_{l} \nabla_{k} \rho_{i j}+\nabla_{h}(R \# R)_{i j k l}\right] \partial_{i} \partial_{j} \partial_{k} \partial_{l} \partial_{h} \text {, } \\
& A_{3}^{j}=0, \quad j \geq 5 \text {. }
\end{aligned}
$$

We note that the operators $A_{0}^{2}, A_{1}^{3}, A_{2}^{4}$, and $A_{3}^{5}$ are zero because of curvature identities. We also compute the following compositions of operators: 


$$
\begin{aligned}
\left.A_{0}^{1} A_{0}\right|_{0} & =(4 / 9)\left[2(\rho * \rho)_{i j}-(R \# \rho)_{i j}\right] \partial_{i} \partial_{j}, \\
A_{0}^{1} A_{0}^{1} & =(4 / 9) \rho_{i j} \rho_{k l} \partial_{i} \partial_{j} \partial_{k} \partial_{l}, \\
\left.A_{0}^{1} A_{1}\right|_{0} & =(1 / 18)\left[-\nabla_{i}\left(\|\rho\|^{2}\right)+12(\rho \# \nabla \rho)_{i}\right] \partial_{i}, \\
\left.A_{0}^{1} A_{1}^{1}\right|_{0} & =(2 / 9)\left[\rho_{i j} \nabla_{k} \tau+4 \nabla_{k}(\rho * \rho)_{i j}-2 \rho_{p q} \nabla_{k} R_{p i q j}\right] \partial_{i} \partial_{j} \partial_{k}, \\
A_{0}^{1} A_{1}^{2} & =(4 / 3) \rho_{i j} \nabla_{h} \rho_{k l} \partial_{i} \partial_{j} \partial_{k} \partial_{l} \partial_{h}, \\
\left.A_{1}^{1} A_{0}\right|_{0} & =(2 / 9)(\rho * \nabla \tau)_{i} \partial_{i}, \\
\left.A_{1}^{1} A_{0}^{1}\right|_{0} & =(2 / 9) \rho_{i j} \nabla_{k} \tau \partial_{i} \partial_{j} \partial_{k}, \\
\left.A_{1}^{2} A_{0}\right|_{0} & =(4 / 3)\left[\nabla_{k}(\rho * \rho)_{i j}+(\rho * \nabla \rho)_{i j k}-R_{p i q j} \nabla_{k} \rho_{p q}-2(R \# \nabla \rho)_{i j k}\right] \partial_{i} \partial_{j} \partial_{k} .
\end{aligned}
$$

Let $B$ be a differential operator with polynomial coefficients. Then $\left.B\right|_{0}$ consists of the terms of $B$ with constant coefficients. Suppose that all terms in $\left.B\right|_{0}$ are of order $k$ such that $\left.B\right|_{0}=b^{i_{1} \ldots i_{k}} \partial_{i_{1}} \cdots \partial_{i_{k}}$. Then define the linear operators $L$ by

$$
L_{k-2 j}^{k}(B)=b^{i_{1} \ldots i_{k}} \sum_{P\left(i_{1} \ldots i_{k}\right)} \frac{\delta_{i_{1} i_{2}} \cdots \delta_{i_{2 j-1} i_{2 j}} \partial_{i_{2 j+1}} \cdots \partial_{i_{k}}}{2^{j} j !(k-2 j) !},
$$

if $j \leq k / 2$. We use the following constants and operators derived from the operators in (5.5) and (5.6) using $L$ :

$$
\begin{aligned}
& L_{0}^{2} A_{0}^{1}=-(2 / 3) \tau, \\
& L_{0}^{2}\left(A_{0}^{1} A_{0}\right)=(4 / 9)\|\rho\|^{2}, \\
& L_{2}^{4}\left(A_{0}^{1} A_{0}^{1}\right)=(8 / 9)\left[\tau \rho_{i j}+2(\rho * \rho)_{i j}\right] \partial_{i} \partial_{j}, \\
& L_{1}^{3}\left(A_{1}^{2}\right)=-4 \nabla_{i} \tau \partial_{i}, \\
&\left.L_{1}^{3}\left(A_{1}^{2}\right) A_{0}\right|_{0}=(8 / 3)(\rho * \nabla \tau)_{i} \partial_{i}, \\
& L_{1}^{3}\left(A_{0}^{1} A_{1}^{1}\right)=(2 / 9)\left[\tau \nabla_{i} \tau+6(\rho * \nabla \tau)_{i}+\nabla_{i}\left(\|\rho\|^{2}\right)+12(\rho \# \nabla \rho)_{i}\right] \partial_{i}, \\
& L_{3}^{5}\left(A_{0}^{1} A_{1}^{2}\right)=(4 / 3)\left[\tau \nabla_{k} \rho_{i j}+2 \rho_{i j} \nabla_{k} \tau+2 \nabla_{k}(\rho * \rho)_{i j}+2(\rho * \nabla \rho)_{i j k}\right] \partial_{i} \partial_{j} \partial_{k}, \\
& L_{1}^{3}\left(A_{1}^{1} A_{0}^{1}\right)=(2 / 9)\left[\tau \nabla_{i} \tau+2(\rho * \nabla \tau)_{i}\right] \partial_{i}, \\
& L_{1}^{3}\left(A_{1}^{2} A_{0}\right)=(2 / 3)\left[4(\rho * \nabla \tau)_{i}+\nabla_{i}\left(\|\rho\|^{2}\right)+4(\rho \# \nabla \rho)_{i}\right] \partial_{i}, \\
& L_{1}^{3}\left(A_{1}^{2}\right) A_{0}^{1}=(8 / 3) \rho_{i j} \nabla_{k} \tau \partial_{i} \partial_{j} \partial_{k}, \\
& L_{1}^{3}\left(L_{1}^{3}\left(A_{1}^{2}\right) A_{0}^{1}\right)=(8 / 3)\left[\tau \nabla_{i} \tau+2(\rho * \nabla \tau)_{i}\right] \partial_{i}, \\
& L_{0}^{2}\left(A_{2}^{2}\right)=-(4 / 45)\left[18 \Delta \tau+2\|\rho\|^{2}+3\|R\|^{2}\right], \\
& L_{2}^{4}\left(A_{2}^{3}\right)=-(8 / 15)\left[27 \nabla_{i} \nabla_{j} \tau+9 \nabla_{p} \nabla_{p} \rho_{i j}-14(R \# \rho)_{i j}\right. \\
&\left.\quad+18(\rho * \rho)_{i j}+6(R * R)_{i j}\right] \partial_{i} \partial_{j},\left.\quad-80(\rho \# \nabla \rho)_{i}-9 \nabla_{i}\left(\|R\|^{2}\right)\right] \partial_{i}, \\
& L_{1}^{3}\left(A_{3}^{3}\right)=(4 / 15)\left[-54 \nabla_{i} \Delta \tau-70(\rho * \nabla \tau)_{i}+14 \nabla_{i}\left(\|\rho\|^{2}\right)\right. \\
& L_{3}^{5}\left(A_{3}^{4}\right)=(32 / 3)\left[-4 \nabla_{k} \nabla_{j} \nabla_{i} \tau-3 \nabla_{k} \nabla_{p} \nabla_{p} \rho_{i j}-6 \nabla_{k}(\rho * \rho)_{i j}\right. \\
&\left.\quad+3 \nabla_{k}(R \# \rho)_{i j}+10(R \# \nabla \rho)_{i j k}-2 \nabla_{k}(R * R)_{i j}\right] \partial_{i} \partial_{j} \partial_{k} .
\end{aligned}
$$


Solutions of Poisson equations at 0 . The procedure described in Section 4 may now be used to express the values of the solutions of (3.6) at 0 . The details of these calculations are too long to include here. We have the following for $u_{i}(0)$ :

$$
\begin{aligned}
u_{1}(0)= & \\
u_{2}(0)= & \frac{\left(A_{0}^{1} u_{0}\right)(0)}{8 n(n+2)}, \\
u_{3}(0)= & \frac{\left(A_{1}^{1} u_{0}\right)(0)}{8 n(n+2)}+\frac{\left(A_{1}^{2} u_{0}\right)(0)}{48 n(n+2)(n+4)}, \\
u_{4}(0)=- & \frac{L_{0}^{2}\left(A_{0}^{1}\right)\left(A_{0}^{1} u_{0}\right)(0)}{16 n^{2}(n+2)(n+4)}+\frac{\left(A_{0}^{1} A_{0} u_{0}+A_{2}^{2} u_{0}\right)(0)}{48 n(n+2)(n+4)} \\
& +\frac{\left(3 A_{0}^{1} A_{0}^{1} u_{0}+A_{2}^{3} u_{0}\right)(0)}{384 n(n+2)(n+4)(n+6)}, \\
u_{5}(0)=- & \frac{L_{0}^{2}\left(A_{0}^{1}\right)\left(A_{1}^{1} u_{0}\right)(0)}{16 n^{2}(n+2)(n+4)}-\frac{L_{0}^{2}\left(A_{0}^{1}\right)\left(A_{1}^{2} u_{0}\right)(0)}{64 n^{2}(n+2)(n+4)(n+6)} \\
& +\frac{\left[(n+8) A_{1}^{1} A_{0} u_{0}-L_{1}^{3}\left(A_{1}^{2}\right) A_{0} u_{0}\right](0)}{48 n(n+2)^{2}(n+4)} \\
& +\frac{\left[3(n+10) A_{1}^{1} A_{0}^{1} u_{0}-4 L_{1}^{3}\left(A_{1}^{2}\right) A_{0}^{1} u_{0}\right](0)}{384 n(n+2)^{2}(n+4)(n+6)} \\
& +\frac{\left(A_{0}^{1} A_{1} u_{0}+A_{3}^{2} u_{0}\right)(0)}{48 n(n+2)(n+4)}+\frac{\left(3 A_{0}^{1} A_{1}^{1} u_{0}+A_{1}^{2} A_{0} u_{0}+A_{3}^{3} u_{0}\right)(0)}{384 n(n+2)(n+4)(n+6)} \\
& +\frac{\left(10 A_{0}^{1} A_{1}^{2} u_{0}+A_{3}^{4} u_{0}\right)(0)}{3840 n(n+2)(n+4)(n+6)(n+8)} .
\end{aligned}
$$

For $v_{i}(0)$ :

$$
\begin{aligned}
& v_{0}(0)=\frac{1}{n} u_{0}(0), \\
& v_{1}(0)=0, \\
& v_{2}(0)=\frac{1}{n} u_{2}(0)-\frac{L_{0}^{2}\left(A_{0}^{1}\right)}{4 n^{2}(n+2)} u_{0}(0),
\end{aligned}
$$




$$
\begin{aligned}
& v_{3}(0)=\frac{1}{n} u_{3}(0)-\frac{L_{0}^{2}\left(A_{0}^{1}\right)}{4 n^{2}(n+2)} u_{1}(0)-\frac{\left[L_{1}^{3}\left(A_{1}^{2}\right) u_{0}-6 A_{1}^{1} u_{0}\right](0)}{24 n(n+2)^{2}(n+4)} \\
& v_{4}(0)=\frac{1}{n} u_{4}(0)-\frac{L_{0}^{2}\left(A_{0}^{1}\right)}{4 n^{2}(n+2)} u_{2}(0) \\
& +\left[\frac{L_{0}^{2}\left(A_{0}^{1}\right) L_{0}^{2}\left(A_{0}^{1}\right)}{8 n^{3}(n+2)(n+4)}-\frac{L_{0}^{2}\left(A_{0}^{1} A_{0}+A_{2}^{2}\right)}{24 n^{2}(n+2)(n+4)}\right] u_{0}(0) \\
& +\frac{\left[6 L_{0}^{2}\left(A_{0}^{1}\right)\left(A_{0}^{1} u_{0}\right)-L_{2}^{4}\left(3 A_{0}^{1} A_{0}^{1}+A_{2}^{3}\right) u_{0}\right](0)}{192 n(n+2)(n+4)^{2}(n+6)} \\
& +\frac{\left(A_{0}^{1} A_{0} u_{0}+A_{2}^{2} u_{0}\right)(0)}{24 n(n+2)(n+4)^{2}(n+6)} \\
& v_{5}(0)=\frac{1}{n} u_{5}(0)-\frac{L_{0}^{2}\left(A_{0}^{1}\right)}{4 n^{2}(n+2)} u_{3}(0) \\
& +\left[\frac{L_{0}^{2}\left(A_{0}^{1}\right) L_{0}^{2}\left(A_{0}^{1}\right)}{8 n^{3}(n+2)(n+4)}-\frac{L_{0}^{2}\left(A_{0}^{1} A_{0}+A_{2}^{2}\right)}{24 n^{2}(n+2)(n+4)}\right] u_{1}(0) \\
& +\frac{L_{0}^{2}\left(A_{0}^{1}\right)\left[(n-6) A_{1}^{1} u_{0}+L_{1}^{3}\left(A_{1}^{2}\right) u_{0}\right](0)}{32 n^{2}(n+2)^{2}(n+4)(n+6)} \\
& +\frac{\left[4 L_{1}^{3}\left(L_{1}^{3}\left(A_{1}^{2} A_{0}^{1}\right) u_{0}-3(n+10) L_{1}^{3}\left(A_{1}^{1} A_{0}^{1}\right) u_{0}\right](0)\right.}{192 n(n+2)^{3}(n+4)(n+6)} \\
& -\frac{\left(3 n^{2}+28 n+68\right)\left[L_{1}^{3}\left(A_{1}^{2}\right) A_{0}^{1} u_{0}\right](0)}{48 n(n+2)^{3}(n+4)^{2}(n+6)^{2}(n+8)} \\
& +\frac{\left.n^{3}+32 n^{2}+244 n+560\right)\left(A_{1}^{1} A_{0}^{1} u_{0}\right)(0)}{64 n(n+2)^{3}(n+4)^{2}(n+6)^{2}(n+8)} \\
& +\frac{L_{0}^{2}\left(A_{0}^{1}\right)\left(A_{1}^{2} u_{0}\right)(0)}{192 n(n+2)(n+4)(n+6)^{2}(n+8)} \\
& -\frac{\left[L_{3}^{5}\left(10 A_{0}^{1} A_{1}^{2}+A_{3}^{4}\right) u_{0}\right](0)}{1920 n(n+2)(n+4)(n+6)^{2}(n+8)} \\
& -\frac{\left(3 n^{2}+28 n+68\right)\left[L_{1}^{3}\left(A_{1}^{2}\right) A_{0}^{1} u_{0}\right](0)}{48 n(n+2)^{3}(n+4)^{2}(n+6)^{2}(n+8)} \\
& +\frac{\left(n^{3}+32 n^{2}+244 n+560\right)\left(A_{1}^{1} A_{0}^{1} u_{0}\right)(0)}{64 n(n+2)^{3}(n+4)^{2}(n+6)^{2}(n+8)} \\
& +\frac{\left(3 A_{0}^{1} A_{1}^{1} u_{0}+A_{1}^{2} A_{0} u_{0}+A_{3}^{3} u_{0}\right)(0)}{192 n(n+2)(n+4)(n+6)^{2}(n+8)} \text {. }
\end{aligned}
$$

Formulas concerning the joint distribution of exit time and place. The first expansion is given in [GP]. 
Proposition 5.1. For smali $\epsilon>0$,

(5.11) $E_{m}[T]=a_{0} \epsilon^{2}+a_{1} \epsilon^{4} \tau_{m}+a_{2} \epsilon^{6}\left\{6 \Delta \tau+(5 / n) \tau^{2}-\|\rho\|^{2}+\|R\|^{2}\right\}_{m}+O\left(\epsilon^{8}\right)$, where $a_{0}, a_{1}$, and $a_{2}$ are positive constants that depend only on the dimension, $n$.

We note that by Corollary 3.2 and Proposition 3.3 , when $u_{0} \equiv 1$,

$$
E_{m}[T]=\epsilon^{2} v_{0}(0)+\epsilon^{3} v_{1}(0)+\epsilon^{4} v_{2}(0)+\epsilon^{5} v_{3}(0)+\epsilon^{6} v_{4}(0)+O\left(\epsilon^{8}\right) .
$$

Substituting the formulas (5.9) and (5.10), we get (5.11) with the following values of $a_{0}, a_{1}$, and $a_{2}$ :

$$
a_{0}=\frac{1}{n}, \quad a_{1}=\frac{1}{6 n^{2}(n+2)}, \quad a_{2}=\frac{1}{90 n^{2}(n+2)(n+4)} .
$$

We give an expansion for $E_{m}\left[\left(\Phi_{\epsilon} f\right)\left(X_{T}\right)\right]$. This extends the results of [L1] and [P2].

Proposition 5.2. Let $f$ be a smooth function on $S$. For small $\epsilon>0$,

$$
\begin{aligned}
& E_{m}\left[\left(\Phi_{\epsilon} f\right)\left(X_{T}\right)\right] \\
& =\int_{S} f(z) d \sigma(z)+b_{1} \epsilon^{2} \int_{S}\left(\rho_{i j}\right)_{m} Q_{i j}(z) f(z) d \sigma(z) \\
& +\epsilon^{3} \int_{S}\left[b_{2}\left(\nabla_{i} \tau\right)_{m} Q_{i}(z)+b_{3}\left(\nabla_{k} \rho_{i j}\right)_{m} Q_{i j k}(z)\right] f(z) d \sigma(z) \\
& +\epsilon^{4} \int_{S}\left\{b _ { 4 } \left[9 \nabla_{i} \nabla_{j} \tau+3 \nabla_{q} \nabla_{q} \rho_{i j}+(10 / n) \tau \rho_{i j}\right.\right. \\
& \left.+6(\rho * \rho)_{i j}-8(R \# \rho)_{i j}+2(R * R)_{i j}\right]_{m} Q_{i j}(z) \\
& \left.+b_{5}\left[18 \nabla_{l} \nabla_{k} \rho_{i j}-5 \rho_{i j} \rho_{k l}+4(R \# R)_{i j k l}\right]_{m} Q_{i j k l}(z)\right\} f(z) d \sigma(z) \\
& +\epsilon^{5} \int_{S}\left\{\left\{b_{6} \tau \nabla_{i} \tau+b_{7}(\rho * \nabla \tau)_{i}\right.\right. \\
& \left.+b_{8}\left[12 \nabla_{i}(\Delta \tau)-7 \nabla_{i}\left(\|\rho\|^{2}\right)+20(\rho \# \nabla \rho)_{i}+2 \nabla_{i}\left(\|R\|^{2}\right)\right]\right\}_{m} Q_{i}(z) \\
& +\left\{b_{9} \tau \nabla_{k} \rho_{i j}+b_{10}(n-2) \rho_{j k} \nabla_{i} \tau+b_{11}\left[12 \nabla_{k} \nabla_{j} \nabla_{i} \tau\right.\right. \\
& +9 \nabla_{k} \nabla_{h} \nabla_{h} \rho_{i j}+18 \nabla_{k}(\rho * \rho)_{i j}-10(\rho * \nabla \rho)_{i j k} \\
& \left.\left.-14 \nabla_{k}(R \# \rho)_{i j}-40(R \# \nabla \rho)_{i j k}+6 \nabla_{k}(R * R)_{i j}\right]\right\}_{m} Q_{i j k}(z) \\
& +b_{12}\left[4 \nabla_{h} \nabla_{l} \nabla_{k} \rho_{i j}-5 \rho_{k l} \nabla_{h} \rho_{i j}\right. \\
& \left.\left.+2 \nabla_{h}(R \# R)_{i j k l}\right]_{m} Q_{i j k l h}\right\} f(z) d \sigma(z) \\
& +O\left(\epsilon^{6}\right) \text {, }
\end{aligned}
$$

where $b_{i}$ are nonzero constants that depend only on the dimension, $n$.

Proof. By Propositions 3.1 and 3.3,

(5.15)

$E_{m}\left[\left(\Phi_{\epsilon} f\right)\left(X_{T}\right)\right]=u_{0}(0)+\epsilon u_{1}(0)+\epsilon^{2} u_{2}(0)+\epsilon^{3} u_{3}(0)+\epsilon^{4} u_{4}(0)+\epsilon^{5} u_{5}(0)+O\left(\epsilon^{6}\right)$

Substituting formulas (5.9) and then using (4.17) we get (5.14) with the following values of $b_{1}, b_{2}, \ldots, b_{12}$ : 


$$
\begin{aligned}
& b_{1}=-\frac{1}{12}, \quad b_{2}=-\frac{1}{24(n+2)}, \quad b_{3}=-\frac{1}{24}, \\
& b_{4}=-\frac{1}{360(n+4)}, \quad b_{5}=-\frac{1}{1440}, \quad b_{6}=-\frac{1}{72 n(n+2)(n+4)}, \\
& b_{7}=-\frac{1}{72(n+2)^{2}}, \quad b_{8}=-\frac{1}{1440(n+2)(n+4)}, \quad b_{9}=-\frac{1}{48 n(n+6)}, \\
& b_{10}=\frac{1}{288(n+2)(n+6)}, \quad b_{11}=-\frac{1}{1440(n+6)}, \quad b_{12}=-\frac{1}{1440} .
\end{aligned}
$$

We give an expansion involving the joint distribution of $T$ and $X_{T}$. This extends the results of [L1].

Proposition 5.3. Let $f$ be a smooth function on $S$. For small $\epsilon>0$,

$$
\begin{aligned}
& E_{m}\left[\begin{array}{l}
\left.T\left(\Phi_{\epsilon} f\right)\left(X_{T}\right)\right]-E_{m}[T] E_{m}\left[\left(\Phi_{\epsilon} f\right)\left(X_{T}\right)\right] \\
=c_{1} \epsilon^{5} \int_{S}\left(\nabla_{i} \tau\right)_{m} Q_{i}(z) f(z) d \sigma(z)
\end{array}\right. \\
& +c_{2} \epsilon^{6} \int_{S}\left[9 \nabla_{i} \nabla_{j} \tau+3 \nabla_{h} \nabla_{h} \rho_{i j}-4(\rho * \rho)_{i j}\right. \\
& \left.\quad+2(R \# \rho)_{i j}+2(R * R)_{i j}\right]_{m} Q_{i j}(z) f(z) d \sigma(z) \\
& +\epsilon^{7} \int_{S}\left\{\begin{array}{l}
\left\{c_{3} \tau \nabla_{i} \tau+c_{4}(\rho * \nabla \tau)_{i}\right. \\
\left.\quad+c_{5}\left[6 \nabla_{i}(\Delta \tau)-\nabla_{i}\left(\|\rho\|^{2}\right)+\nabla_{i}\left(\|R\|^{2}\right)\right]\right\}_{m} Q_{i}(z)
\end{array}\right. \\
& +\begin{array}{r}
\left\{c_{6} \rho_{j k} \nabla_{i} \tau+c_{7}\left[4 \nabla_{k} \nabla_{j} \nabla_{i} \tau+3 \nabla_{k} \nabla_{h} \nabla_{h} \rho_{i j}-4 \nabla_{k}(\rho * \rho)_{i j}\right.\right. \\
\left.\left.\left.\quad+2 \nabla_{k}(R \# \rho)_{i j}+2 \nabla_{k}(R * R)_{i j}\right]\right\}_{m} Q_{i j k}(z)\right\} f(z) d \sigma(z)
\end{array} \\
& +O\left(\epsilon^{8}\right), \quad
\end{aligned}
$$

where $c_{1}, c_{2}, \ldots, c_{7}$ are nonzero constants that depend only on the dimension, $n$.

Proof. As before, we use Propositions 3.1 and 3.3. We then substitute formula (5.10) and, using (4.17), we get (5.17) with constants

$$
\begin{array}{ll}
c_{1}=\frac{1}{12(n+2)^{2}(n+4)}, & c_{2}=\frac{1}{360(n+4)^{2}(n+6)}, \\
c_{3}=\frac{5 n+6}{72 n(n+2)^{3}(n+4)(n+6)}, & c_{4}=-\frac{1}{6(n+2)^{3}(n+4)(n+6)}, \\
c_{5}=\frac{1}{144(n+2)^{2}(n+4)(n+6)}, & c_{6}=-\frac{1}{144(n+2)^{2}(n+4)}, \\
c_{7}=\frac{1}{720(n+6)^{2}(n+8)} . &
\end{array}
$$

\section{Curvature conditions}

Asymptotic mean value condition. We now use Proposition 5.2 to derive curvature conditions for manifolds satisfying the asymptotic mean value condition 
(AMVC). Recall that for positive integer $k$ AMVC is defined as follows:

$$
E_{m}\left[f\left(\exp _{m}^{-1}\left(X_{T}\right) / \epsilon\right)\right]=\int_{S} f(z) d \sigma(z)+o\left(\epsilon^{k}\right), \quad \epsilon \downarrow 0,
$$

for every $f$, a smooth function on $S$, and for all $m \in M$.

Theorem 6.1, below, gives relationships between AMVC and the curvature conditions (expressed in normal coordinates at $m$ ):

$$
\begin{gathered}
\sum_{P(p q r s)}\left[(R \# R)_{p q r s}-\frac{2\|\rho\|^{2}+3\|R\|^{2}}{2 n(n+2)} g_{p q} g_{r s}\right]_{m}=0, \quad 1 \leq p, q, r, s \leq n, \\
{\left[\nabla_{p}\left(\|R\|^{2}\right)\right]_{m}=0, \quad 1 \leq p \leq n .}
\end{gathered}
$$

Theorem 6.1. A Riemannian manifold $M$ and Brownian motion $X$ on $M$ satisfy $A M V C$

(1) for $k=2$ if and only if (6.1a) holds for every $m \in M$

(2) for $k=3$ if and only if $(6.1 \mathrm{a}-\mathrm{b})$ hold for every $m \in M$;

(3) for $k=4$ if and only if $(6.1 \mathrm{a}-\mathrm{c})$ hold for every $m \in M$;

(4) for $k=5$ if and only if (6.1a-d) hold for every $m \in M$.

Proof. As noted in Section 2, it is sufficient to consider only functions of the form $f(z)=Q_{p_{1} \ldots p_{k}}(z)$. Letting $f(z)=Q_{p q}(z)$ in the $\epsilon^{2}$ term of (5.14) and applying Lemma 4.7, part (1) follows.

To prove part (2), letting $f(z)=Q_{p}(z)$ in the $\epsilon^{3}$ term of (5.14) and applying Lemma 4.7, we have that AMVC for $k=3$ also implies (6.1b) for $m \in M$. We note that $(6.1 \mathrm{a}-\mathrm{b})$ imply

$$
\left(\nabla_{r} \rho_{p q}\right)_{m}=0, \quad 1 \leq p, q, r \leq n .
$$

Thus the converse also follows.

To prove part (3), we first note that if $(6.1 \mathrm{a}-\mathrm{b})$ hold for every $m \in M$,

$$
\nabla \tau=0=\nabla \rho .
$$

Letting $f(z)=Q_{p q}(z)$ in the $\epsilon^{4}$ term of (5.14), applying Lemma 4.7, and using (6.3), we have that AMVC for $k=4$ implies

$$
\begin{gathered}
{\left[(5 / n) \tau \rho_{p q}+3(\rho * \rho)_{p q}-4(R \# \rho)_{p q}+(R * R)_{p q}\right]_{m}} \\
=(1 / n)\left[\left((5 / n) \tau^{2}-\|\rho\|^{2}+\|R\|^{2}\right) g_{p q}\right]_{m} .
\end{gathered}
$$

Using (6.1a), this reduces to

$$
\left[(R * R)_{p q}\right]_{m}=(1 / n)\left[\|R\|^{2} g_{p q}\right]_{m}, \quad 1 \leq p, q \leq n .
$$


Now letting $f(z)=Q_{p q r s}(z)$ in the $\epsilon^{4}$ term of (5.14), applying Lemma 4.7, and using (6.3), we have that AMVC for $k=4$ implies

$$
\begin{aligned}
& \sum_{P(p q r s)}\left\{4(R \# R)_{p q r s}-5 \rho_{p q} \rho_{r s}\right. \\
&-\frac{1}{n+4}\left[12(R * R)_{r s}+8(R \# \rho)_{r s}-20(\rho * \rho)_{r s}-10 \tau \rho_{r s}\right] g_{p q} \\
&\left.+\frac{1}{(n+2)(n+4)}\left[6\|R\|^{2}-6\|\rho\|^{2}-5 \tau^{2}\right] g_{p q} g_{r s}\right\}_{m}=0 .
\end{aligned}
$$

Using (6.1a) and (6.5), this reduces to (6.1c).

To prove the converse, we note that that (6.1a) and (6.1c) imply (6.5) [B, pp. 164-165] and thus also (6.6). If (6.1a-b) hold for every $m \in M$ then (6.3) also holds. Therefore if $(6.1 \mathrm{a}-\mathrm{c})$ hold for every $m \in M$, AMVC holds for $k=4$.

Finally, to prove part (4), letting $f(z)=Q_{p}(z)$ in the $\epsilon^{5}$ term of (5.14), applying Lemma 4.7, and using (6.3), we have that AMVC for $k=5$ implies (6.1d) for every $m \in M$. Conversely, from (6.1a-d), (6.3), and (6.5) it follows that all parts of the $\epsilon^{5}$ term of (5.14) vanish. Therefore AMVC holds for $k=5$ if $(6.1 \mathrm{a}-\mathrm{d})$ hold for every $m \in M$.

Asymptotic uncorrelated condition. Proceeding as above, we use Proposition 5.3 to derive curvature conditions for manifolds satisfying the asymptotic uncorrelated condition (AUC). Recall that for positive integer $k$ AUC is defined as follows:

$$
E_{m}\left[T f\left(\exp _{m}^{-1}\left(X_{T}\right) / \epsilon\right)\right]-E_{m}[T] E_{m}\left[f\left(\exp _{m}^{-1}\left(X_{T}\right) / \epsilon\right)\right]=o\left(\epsilon^{k+2}\right), \quad \epsilon \downarrow 0,
$$

for every $f$, a smooth function on $S$, and for all $m \in M$.

Theorem 6.2 gives relationships between AUC and the curvature conditions (expressed in normal coordinates at $m$ ):

$$
\left(\nabla_{p} \tau\right)_{m}=0, \quad 1 \leq p \leq n
$$

$$
\begin{aligned}
& {\left[3 \nabla_{h} \nabla_{h} \rho_{p q}-4(\rho * \rho)_{p q}+\right.} 2(R \# \rho)_{p q}+2(R * R)_{p q} \\
&\left.-\frac{-2\|\rho\|^{2}+2\|R\|^{2}}{n} g_{p q}\right]_{m}=0, \\
& 1 \leq p, q \leq n,
\end{aligned}
$$

$$
\left[\nabla_{p}\left(\|R\|^{2}-\|\rho\|^{2}\right)\right]_{m}=0, \quad 1 \leq p \leq n .
$$

These conditions are overall weaker than the conditions $(6.1 \mathrm{a}-\mathrm{d})$.

Theorem 6.2. A Riemannian manifold $M$ and Brownian motion $X$ on $M$ satisfy $A U C$

(1) for $k=3$ if and only if (6.7a) holds for every $m \in M$;

(2) for $k=4$ if and only if $(6.7 \mathrm{a}-\mathrm{b})$ hold for every $m \in M$;

(3) for $k=5$ if and only if $(6.7 \mathrm{a}-\mathrm{c})$ hold for every $m \in M$. 
Proof. As before, we only consider functions of the form $f(z)=Q_{p_{1} \ldots p_{k}}(z)$. Letting $f(z)=Q_{p}(z)$ in the $\epsilon^{5}$ term of (5.17) and applying Lemma 4.7, part (1) follows. We note that if $(6.7 \mathrm{a})$ holds for every $m \in M$ or, equivalently, if AUC holds for $k \geq 3$, then $\tau$ is constant. Using this fact, letting $f(z)=Q_{p q}(z)$ in the $\epsilon^{6}$ term of (5.17), and applying Lemma 4.7, part (2) follows.

Finally, to prove part (3), letting $f(z)=Q_{p}(z)$ in the $\epsilon^{7}$ term of (5.17), applying Lemma 4.7, and using the fact that $\tau$ is constant, we have that AUC for $k=5$ implies $(6.7 \mathrm{c})$ for every $m \in M$. Conversely, if $(6.7 \mathrm{a}, \mathrm{c})$ hold for every $m \in M, \tau$ and $\|R\|^{2}-\|\rho\|^{2}$ are constant. It follows from (6.7b) that

$$
\begin{array}{r}
\left\{\nabla_{r}\left[3 \nabla_{h} \nabla_{h} \rho_{p q}-4(\rho * \rho)_{p q}+2(R \# \rho)_{p q}+2(R * R)_{p q}\right]\right\}_{m}=0, \\
1 \leq p, q, r \leq n .
\end{array}
$$

Therefore, all parts of the $\epsilon^{7}$ term of (5.17) vanish and thus AUC holds for $k=5$ if $(6.7 \mathrm{a}-\mathrm{c})$ hold for every $m \in M$.

The first statement below follows immediately since the independence of $T$ and $X_{T}$ implies AUC for all positive integers $k$. The second statement follows applying the condition (6.1a) to $(6.7 \mathrm{~b}-\mathrm{c})$.

Corollary 6.3. If $T$ and $X_{T}$ are independent random variables for small positive values of $\epsilon$, then conditions $(6.7 \mathrm{a}-\mathrm{c})$ hold for every $m \in M$. If in addition, $M$ is an Einstein manifold, condition (6.5) holds for every $m \in M$ and $\|R\|^{2}$ is constant on $M$.

\section{REMARKS}

These results can be extended in the following ways. First, the reduction procedure given in Section 4 can be applied to the solutions of larger systems of Poisson equations of the same form as (3.6):

$$
\begin{aligned}
\Delta_{-2} u_{0}^{(0)} & =0 \text { in } B, \quad u_{0}^{(0)}=f \text { on } S, \\
\Delta_{-2} u_{j}^{(0)}+\sum_{i=2}^{j} \Delta_{i-2} u_{j-i}^{(0)} & =0 \text { in } B, \quad u_{j}^{(0)}=0 \text { on } S \quad(1 \leq j \leq k), \\
\Delta_{-2} u_{j}^{(1)}+\sum_{i=2}^{j} \Delta_{i-2} u_{j-i}^{(1)}+2 u_{j}^{(0)} & =0 \text { in } B, \quad u_{j}^{(1)}=0 \text { on } S \quad(0 \leq j \leq k), \\
\vdots & \\
\Delta_{-2} u_{j}^{(l)}+\sum_{i=2}^{j} \Delta_{i-2} u_{j-i}^{(l)}+2 u_{j}^{(l-1)} & =0 \text { in } B, \quad u_{j}^{(l)}=0 \text { on } S \quad(0 \leq j \leq k) .
\end{aligned}
$$

Then asymptotic expansions of $E_{m}\left[T^{l}\left(\Phi_{\epsilon} f\right)\left(X_{T}\right)\right]$ in $\epsilon$ can be computed as

$$
E_{m}\left[T^{l}\left(\Phi_{\epsilon} f\right)\left(X_{T}\right)\right]=l ! \sum_{j=0}^{k} \epsilon^{j+2 l} u_{j}^{(l)}(0)+O\left(\epsilon^{k+2 l+1}\right) .
$$

Additional terms in the asymptotic expansions (5.11), (5.14), and (5.17) can be computed provided one computes enough terms in the homogeneous decomposition of the Laplacian. It is possible, in principle, to compute any term of the 
expansion in $\epsilon$ of $E_{m}\left[T^{l}\left(\Phi_{\epsilon} f\right)\left(X_{T}\right)\right]$. In practice, the size of this task increases extremely rapidly as either $l$ or the level of asymptotics increases.

Finally, these methods can be extended to any diffusion with infinitesimal generator $A$ which admits a homogeneous decompostion with respect to normal coordinates $\left(x^{j}\right)$ :

$$
A=\frac{1}{2} \Delta_{-2}+\frac{1}{2} \sum_{i=-1}^{\infty} A_{i},
$$

where $\Delta_{-2}$ is the ordinary Euclidean Laplacian and $A_{i}$ is a second order linear differential operator with polynomial coefficients of the form $A_{i}=q_{i}^{j} \partial_{j}+$ $q_{i}^{j k} \partial_{j} \partial_{k}$ where $q_{i}^{j}$ and $q_{i}^{j k}$ are respectively $(i+1)$-degree and $(i+2)$-degree homogeneous polynomials in $\left(x^{j}\right)$.

We also note that due to the large number of calculations involved, the calculations leading to all terms in (5.11), all terms except the $\epsilon^{5}$ term in (5.14), and all terms except the $\epsilon^{7}$ term in (5.17) were first computed by hand and then checked using computer symbolic algebra programs. The last term in each of (5.14) and (5.17) is based in part on computations made by computer. The programs used in these computations were Mathematica(TM), Version 2.0, a mathematical software system developed and distributed by Wolfram Research, Inc., and MathTensor(TM), a Mathematica-based tensor analysis program developed and distributed by Leonard Parker and Steven Christensen, founders of MathSolutions, Inc.

\section{REFERENCES}

[B] A. L. Besse, Manifolds all of whose geodesics are closed, Ergeb. Math. Grenzgeb., vol. 93, Springer, Berlin, 1978.

[CH] R. Courant and D. Hilbert, Methods of mathematical physics, vol. 2, Interscience, New York, 1962.

[DR] E. Damek and F. Ricci, Harmonic analysis on solvable extensions of H-type groups, J. Geom. Anal. 2 (1992), 213-248.

[GP] A. Gray and M. Pinsky, The mean exit time from a small geodesic ball in a Riemannian manifold, Bull. Sci. Math. (2) 107 (1983), 345-370.

[Hu] H. R. Hughes, Brownian exit distributions from normal balls in $S^{3} \times H^{3}$, Ann. Probab. 20 (1992), 655-659.

[IW] N. Ikeda and S. Watanabe, Stochastic differential equations and diffusion processes, 2nd ed., North-Holland, Amsterdam, 1989.

[IM] K. Itô and H. P. McKean, Jr., Diffusion processes and their sample paths, Grundlehren Math. Wiss., 125, Springer, Berlin, 1965.

[K] O. Kowalski, The second mean-value operator on Riemannian manifolds, Proceedings of the CSSR-GDR-Polish conference on differential geometry and its applications, Nové Město 1980, Universita Karlova Praha, 1982, pp. 33-45.

[KO1] M. Kozaki and Y. Ogura, On geometric and stochastic mean values for small geodesic spheres in Riemannian manifolds, Tsukuba J. Math. 11 (1987), 131-145.

[KO2] _ On the independence of exit time and exit position from small geodesic balls for Brownian motions on Riemannian manifolds, Math. Z. 197 (1988), 561-581.

[L1] M. Liao, Hitting distributions of small geodesic spheres, Ann. Probab. 16 (1988), 10391050.

[L2] _ An independence property of Riemannian Brownian motions, Geometry of Random Motion, Contemp. Math., vol. 73, Amer. Math. Soc., Providence, RI, 1988, pp. 197-201. 
[P1] M. Pinsky, Moyenne stochastique sur une variette riemannienne, C. R. Acad. Sci. Paris 292 (1981), 991-994.

[P2] - On non-Euclidean harmonic measure, Ann. Inst. H. Poincaré 21 (1985), 39-46.

[RWW] H. Ruse, H. G. Walker, and T. J. Willmore, Harmonic spaces, Monogr. Mat., 8, Edizioni Cremonese, Roma, 1961.

[VK] N. Ja. Vilenkin and A. U. Klimyk, Representation of Lie groups and special functions, Volume 2: class I representations, special functions, and integral transforms, Mathematics and its Applications (Soviet Series), vol. 74, Kluwer, Dordrecht, 1993.

Department of Mathematics, Southern Illinois University, Carbondale, IL 62901

E-mail address: hrhughes@c-math1.siu.edu 